\title{
MR Imaging Features of Middle Cranial Fossa Encephaloceles and Their Associations with Epilepsy
}

\author{
(D) D.R. Pettersson, (D) K.S. Hagen, (D) N.C. Sathe, (D)B.D. Clark, and (D)D.C. Spencer
}

\begin{abstract}
BACKGROUND AND PURPOSE: Middle cranial fossa encephaloceles are an increasingly recognized cause of epilepsy; however, they are also often encountered on neuroimaging in patients with no history of seizure. We characterized the MR imaging features of middle cranial fossa encephaloceles in seizure and nonseizure groups with the hope of uncovering features predictive of epileptogenicity.
\end{abstract}

MATERIALS AND METHODS: Seventy-seven patients with middle cranial fossa encephaloceles were prospectively identified during routine clinical practice of neuroradiology at a tertiary care hospital during an 18-month period. Thirty-five of 77 (45\%) had a history of seizure, 20/77 (26\%) had temporal lobe epilepsy, and 42/77 (55\%) had no history of seizures. Middle cranial fossa encephalocele features on MR imaging were characterized, including depth, area, number, location, presence of adjacent encephalomalacia, and degree of associated parenchymal morphologic distortion. MR imaging features were compared between the seizure and nonseizure groups.

RESULTS: No significant difference in MR imaging features of middle cranial fossa encephaloceles was seen when comparing the seizure and nonseizure groups. Comparison of just those patients with temporal lobe epilepsy $(n=20)$ with those with no history of seizure $(n=42)$ also found no significant difference in MR imaging features.

CONCLUSIONS: Anatomic MR imaging features of middle cranial fossa encephaloceles such as size, number, adjacent encephalomalacia, and the degree of adjacent parenchymal morphologic distortion may not be useful in predicting likelihood of epileptogenicity.

ABBREVIATIONS: MCF = middle cranial fossa; MCFE = middle cranial fossa encephalocele; TLE = temporal lobe epilepsy

D uring the past decade, awareness of aberrant arachnoid granulations and encephaloceles occurring in the middle cranial fossa (MCF) has grown substantially. As of 2010, only 12 cases of middle cranial fossa encephaloceles (MCFEs) had been reported in the literature, ${ }^{1}$ and several times that amount has been reported since. ${ }^{2-4}$ Patients with encephaloceles involving the sphenoid sinus or temporal bone often come to the attention of otolaryngologists and neurosurgeons when they present with a CSF leak or meningitis. ${ }^{2,5,6}$ Patients with MCFEs can come to the

Received May 29, 2020; accepted after revision July 24.

From the Departments of Radiology (D.R.P., B.D.C.), Neurology (K.S.H., D.C.S.), and School of Medicine (N.C.S.), Oregon Health \& Science University, Portland, Oregon.

Preliminary data previously presented at: American Epilepsy Society Annual Meeting, December 6-10, 2019; Baltimore, Maryland.

Please address correspondence to David R. Pettersson, MD, Oregon Health \& Science University, 3181 SW Sam Jackson Park Rd, Portland, OR, 97239; e-mail: petterss@ohsu.edu

Indicates article with supplemental on-line tables.

Indicates article with supplemental on-line photo.

http://dx.doi.org/10.3174/ajnr.A6798 attention of epileptologists when they present with seizures.? However, recent work in the radiology literature by Benson et $\mathrm{al}^{3}$ has established that MCFEs and arachnoid pits are common asymptomatic incidental findings on neuroimaging using highresolution T2-weighted imaging. They reviewed 203 consecutive internal auditory canal MR imaging examinations and found MCF arachnoid pits (defects not containing brain parenchyma) in $22 \%$ of patients and MCFEs (defects containing brain parenchyma) in another $5 \%$ of patients, none of whom had a history of seizure. Campbell et $\mathrm{al}^{8}$ investigated the prevalence of MCFEs in 418 patients with refractory epilepsy and found that MCFEs were present in $12 \%$ of patients, and they were found more frequently in patients with temporal lobe epilepsy (TLE) than in patients without it. Last, there are several case reports and small case series describing patients with TLE and MCFEs who become seizurefree following resection or disconnection of the cerebral tissue in the MCFE, suggesting a causative association between some MCFEs and epilepsy. ${ }^{7,9-14}$

Thus, these recent works have demonstrated that MCFEs should no longer be considered rare, are often asymptomatic (ie, 
do not cause seizures), and can be the cause of seizures in some patients with epilepsy. These observations can pose a diagnostic dilemma when an MCFE is identified on MR imaging when evaluating a patient with drug-resistant epilepsy for possible surgery. In that scenario, it may be unclear whether the MCFE is the etiology of the epilepsy. While scalp electrographic and semiologic features may help to clarify the relevance of a given MCFE, it remains unclear whether there are specific anatomic MR imaging features of MCFEs associated with epileptogenicity. In the work presented here, we sought to address that knowledge gap by comparing the MR imaging features of MCFEs between 2 groups of patients: those with a history of seizure and those without.

\section{MATERIALS AND METHODS}

\section{Patient Selection}

Institutional review board approval was obtained for this study. A neuroradiologist with a Certificate of Additional Qualification in the subspecialty of Neuroradiology from the American Board of Radiology, working at a tertiary care teaching hospital, made note of every case of MCFE prospectively encountered during routine clinical neuroradiology practice during an 18-month period. A minority of cases were encountered while reviewing imaging for a fortnightly adult epilepsy multidisciplinary conference. Patients were not excluded on the basis of age, clinical history, type of MR imaging examination, or other findings seen on imaging. Cases were included only if the MCF defect definitively contained brain parenchyma; defects containing only CSF (ie, arachnoid pits, meningoceles) and no brain parenchyma were not included. MCFEs involving the mastoid or petrous portions of the temporal bone or tegmen tympani were also excluded.

\section{Imaging Review}

After all cases were collected, the PACS account for each patient was accessed. All available brain MR imaging for each patient was reviewed by the certificated neuroradiologist with the intent of identifying the MR imaging examination and sequences best depicting the MCFE to use for additional imaging review. The type and section thickness of the most informative sequence for each patient were recorded. Additional sequences were used to help with MCFE localization and measurement, typically in planes orthogonal to the primary sequence used for the image review.

Length measurements were made on images from those sequences using the tools embedded in the PACS. A single neuroradiologist with 5 years of post-neuroradiology fellowship experience reviewed each case, making note of MCFE laterality (right, left, bilateral) and number $(1,2,3,4,5$, and $>5)$. For each patient, additional measurements were made of the largest single encephalocele present on the scan. These measurements included the 3D size of the osseous defect ( 3 orthogonal dimensions by caliper measurement, aligned to the plane of the inner table of the calvaria), volume (using the formula for a hemi-ellipsoid, approximating the scalloped morphology of the defects), location (sextant divisions of each MCF, anterior to the mastoid and petrous portions of the temporal bone), degree of brain parenchymal morphologic distortion in or adjacent to the MCFE (subjective 4-point Likert scale of minimal, mild, moderate, and severe distortion; and caliper measurement of the longest single dimension of definitively distorted brain parenchyma), the presence of encephalomalacia (increased T2 signal and volume loss) of the brain parenchyma in or immediately adjacent to the MCFE, and the presence of punctate or linear CSFintensity $\mathrm{T} 2$ signal within the brain parenchyma in the MCFE. The presence or absence of a subjective asymmetric increase of the intracranial subarachnoid space volume adjacent to the encephalocele was also noted. MCF sextant boundaries (On-line Figure) were approximated by dividing the transverse dimension into equallength thirds (medial, mid, lateral) and dividing the anterior-posterior dimension into equal halves (polar/anterior, floor/posterior), excluding the petrous and mastoid portions of the temporal bone. The radiologist was not blinded to patient history during the imaging review.

Of the 77 patients included in the study, the primary MR imaging sequences used for the imaging review were $2 \mathrm{D}$ STIR $(n=37)$, 2D T2 TSE $(n=14), 3 \mathrm{D}$ T2 gradient-based sequences $(n=15), 3 \mathrm{D}$ T2 FSE $(n=8)$, 2D FLAIR $(n=2)$, and 3D FLAIR $(n=1)$. The section thicknesses of the primary sequences used for imaging review were $0.6-1.2 \mathrm{~mm}(n=18), 2 \mathrm{~mm}(n=31), 3 \mathrm{~mm}(n=15)$, $4 \mathrm{~mm}(n=12)$, and $5 \mathrm{~mm}(n=1)$. The types of MR imaging protocols of the studies used for the image review included epilepsy $(n=$ $27)$, routine brain $(n=25)$, ear, nose, and throat $(n=12)$, internal auditory canal $(n=9)$, and stereotactic brain protocols $(n=4)$. The magnetic field strength of the scans used for the imaging review included 3T $(n=49), 1.5 \mathrm{~T}(n=27)$, and $1 \mathrm{~T}(n=1)$.

\section{Clinical Review}

Electronic medical records of all patients were retrospectively reviewed by a fellowship-trained epileptologist for any history of seizure or epilepsy, as defined by the International League Against Epilepsy. ${ }^{15}$ For those with a history of seizure or epilepsy, all available diagnostic and clinical data were reviewed and patients were categorized by type of epilepsy (TLE, non-TLE, unknown), predominant laterality of seizures (left hemisphere, right hemisphere, bilateral, or unknown), presence of drug-resistant epilepsy at the time of most recent clinical note, and presence of additional seizure risk factors. Of the 35 patients with a diagnosis of either seizure or epilepsy, the medical record included clinical notes from an epileptologist $(n=24)$, clinical notes from a general neurologist $(n=4)$, an epilepsy-monitoring unit admission $(n=20)$, scalp electroencephalogram $(n=31)$, video-electroencephalogram $(n=6)$, stereotactic electroencephalogram $(n=2)$, foramen ovale electrodes $(n=1)$, brain PET scan $(n=$ $5)$, brain SPECT $(n=1)$, or none of the above $(n=3)$.

\section{Statistical Analysis}

The Pearson $\chi^{2}$ test or Fisher exact test was used to examine the statistical significance of categoric MR imaging characteristics of MCFEs between the group of patients with no history of seizures and the group of patients with a history of seizures. The Wilcoxon rank sum test, also known as the Mann-Whitney test, was used to assess whether the difference in the medians of continuous MR imaging characteristics of MCFEs were statistically significantly different between the seizure and nonseizure groups. Some categoric imaging characteristics such as the MCF sextants containing the largest MCFE and the number of bilateral MCFEs were further collapsed to maintain adequate cell sizes. We conducted a sensitivity 
Table 1: MR imaging characteristics of encephaloceles compared between patients with a history of seizure and those with no history of seizure ${ }^{a}$

\begin{tabular}{|c|c|c|c|c|}
\hline Variable & $\begin{array}{c}\text { Total } \\
(n=77)\end{array}$ & $\begin{array}{l}\text { History of Seizure } \\
\quad(n=35)\end{array}$ & $\begin{array}{l}\text { No History of } \\
\text { Seizure }(n=42)\end{array}$ & $P$ \\
\hline Location of encephaloceles & & & & .91 \\
\hline Left & $22(28.6)$ & $10(28.6)$ & $12(28.6)$ & \\
\hline Right & $16(20.8)$ & $8(22.9)$ & 8 (19.1) & \\
\hline Bilateral & $39(50.7)$ & $17(48.6)$ & $22(52.4)$ & \\
\hline Side of largest encephalocele & & & & .80 \\
\hline Left & $43(55.8)$ & $19(54.3)$ & $24(57.1)$ & \\
\hline Right & $34(44.2)$ & $16(45.7)$ & $18(42.9)$ & \\
\hline Total No. of encephaloceles & & & & .12 \\
\hline 1 & $26(33.8)$ & $15(42.9)$ & $11(26.2)$ & \\
\hline$>1$ & 51 (66.2) & $20(57.1)$ & $31(73.8)$ & \\
\hline $\begin{array}{l}\text { Median No. of sextants }{ }^{\mathrm{b}} \text { containing encephaloceles } \\
\text { Largest encephalocele }\end{array}$ & $3(2)$ & $2(2)$ & $3(3)$ & .21 \\
\hline Depth (mm) & $5(3)$ & $5(3)$ & $5.5(3)$ & .88 \\
\hline Areal extent $\left(\mathrm{mm}^{2}\right)$ & $117(142)$ & $119(144)$ & $117(154)$ & .93 \\
\hline Volume $\left(\mathrm{mm}^{3}\right)$ & $368(538)$ & $374(512)$ & $364(632)$ & .76 \\
\hline Morphologic distortion of adjacent brain parenchyma & & & & .51 \\
\hline Minimal & $11(14.3)$ & $4(11.4)$ & $7(16.7)$ & \\
\hline Mild & $21(27.3)$ & $10(28.6)$ & $11(26.2)$ & \\
\hline Moderate & $32(41.6)$ & $17(48.6)$ & $15(35.7)$ & \\
\hline Severe & $13(16.9)$ & $4(11.4)$ & $9(21.4)$ & \\
\hline $\begin{array}{l}\text { Longest single dimension of distorted parenchyma adjacent to largest } \\
\text { encephalocele (mm) }\end{array}$ & $13(11)$ & $14(13)$ & $13(11)$ & .54 \\
\hline Presence of adjacent encephalomalacia & 9 (11.7) & $2(5.7)$ & $7(16.7)$ & .14 \\
\hline
\end{tabular}

${ }^{a}$ Data are median (interquartile range) or No. (\%)

${ }^{\mathrm{b}}$ Middle cranial fossa sextants.

analysis of only those that had TLE and compared MR imaging characteristics of MCFE in this group with the those in the nonseizure group using the same methods as described above. Finally, we descriptively examined seizure characteristics among epileptics. All analyses were performed using STATA, Version 15 (StataCorp, 2017; Stata Statistical Software: Release 15).

\section{RESULTS}

\section{MR Imaging Findings}

Seventy-seven patients with MCFEs were included in the study (Table 1 and On-line Table 1). MCFEs were seen bilaterally in $39 / 77$ (51\%), only on the left in $22 / 77$ (29\%), and only on the right in 16/77 (21\%). Twenty-six of 77 (34\%) had only a solitary MCFE, 29/77 (38\%) had between 2 and 5 MCFEs, and the remaining 22/77 (29\%) had >5 MCFEs. Regarding the largest MCFE present in each patient, the most common location was the medial polar/anterior sextant $(41 / 77,53 \%)$ followed by the mid-polar/anterior sextant $(19 / 77,25 \%)$, with the remainder of encephaloceles located in the lateral and inferior/floor portions of the MCF. The largest MCFE present in each patient had a mean depth of $5 \mathrm{~mm}$ (perpendicular to the inner table of calvaria), a mean area of $117 \mathrm{~mm}^{2}$, and a mean volume of $368 \mathrm{~mm}^{3}$. The degree of brain parenchymal distortion in and adjacent to the largest MCFE was subjectively categorized as minimal (14\%), mild $(27 \%)$, moderate $(42 \%)$, or severe $(17 \%)$. The mean longest single dimension of definitively distorted parenchyma within or adjacent to the largest MCFE was $13 \mathrm{~mm}$. Encephalomalacia (parenchymal T2 hyperintensity and subjective volume loss) in or adjacent to the largest MCFE was present in only $12 \%$ of patients. The intracranial subarachnoid space adjacent to the largest MCFE was asymmetrically enlarged in $38 \%$ of patients. Last, foci of CSF-intensity T2 signal were seen in the parenchyma within the largest encephalocele in $53 \%$ of patients.

\section{Clinical Findings}

The average patient age at the time of medical record review was 53 years, with an absolute range of 9-96 years and an interquartile range of 33-62 years. Forty-nine of 77 (64\%) of patients were female. Of the 77 patients included in the study, 42 (55\%) had no history of seizure and 35 (45\%) had a history of seizure or epilepsy. Twenty-six of 35 (74\%) patients with a history of seizure had a risk factor for seizure other than MCFE. TLE was the most common epilepsy type $(20 / 35,57 \%)$, followed by non-TLE (11/ $35,31 \%)$ and unknown epilepsy or seizure type $(4 / 35,11 \%)$. For patients with a history of seizure, seizure laterality was left hemisphere $(16 / 35,46 \%)$, right hemisphere $(7 / 35,20 \%)$, bilateral $(5 / 35,14 \%)$, and unknown laterality $(7 / 35,20 \%)$.

\section{Associations}

There were no statistically significant differences in the conventional MR imaging features of MCFEs between those patients with a history of seizure and those without (Table 1). Encephalomalacia of the brain parenchyma located within or adjacent to the MCFE was seen almost 3 times more frequently in the nonseizure group (7/42, $17 \%)$ compared with the seizure group $(2 / 35,6 \%)$, though this did not meet statistical significance $(P=.14)$. The mean volume of the largest MCFE found in each patient also showed no significant difference between the seizure $\left(374 \mathrm{~mm}^{2}\right)$ and nonseizure $\left(364 \mathrm{~mm}^{2}\right)$ groups $(P=.76)$. The subjective degree of brain parenchymal 
Table 2: MR imaging characteristics of middle cranial fossa encephaloceles compared in patients with a history of temporal lobe epilepsy and those with no history of seizure ${ }^{a}$

\begin{tabular}{|c|c|c|c|c|}
\hline Variable & $\begin{array}{c}\text { Total } \\
(n=62)\end{array}$ & $\begin{array}{l}\text { History of Temporal Lobe } \\
\text { Epilepsy }(n=20)\end{array}$ & $\begin{array}{l}\text { No History of } \\
\text { Seizure }(n=42)\end{array}$ & $P$ \\
\hline Encephalocele side & & & & .86 \\
\hline Left & $17(27.4)$ & $5(25)$ & $12(28.6)$ & \\
\hline Right & $30(21.0)$ & $5(25)$ & $8(19.1)$ & \\
\hline Bilateral & $32(51.6)$ & $10(50)$ & $22(52.4)$ & \\
\hline Side of largest encephalocele & & & & .60 \\
\hline Left & $34(54.8)$ & $10(50.0)$ & $24(57.1)$ & \\
\hline Right & $28(45.2)$ & $10(50.0)$ & $18(42.9)$ & \\
\hline Total No. of bilateral encephaloceles & & & & .14 \\
\hline 1 & $20(32.3)$ & $9(45.0)$ & $11(26.2)$ & \\
\hline$>1$ & $42(67.7)$ & $11(55.0)$ & $31(73.8)$ & \\
\hline $\begin{array}{l}\text { Median No. of sextants }{ }^{\mathrm{b}} \text { containing encephaloceles } \\
\text { Largest encephalocele }\end{array}$ & $3(2)$ & $3(2)$ & $3(3)$ & .23 \\
\hline Depth $(\mathrm{mm})$ & $5(3)$ & $4(2.5)$ & $5.5(3)$ & .43 \\
\hline Areal extent $\left(\mathrm{mm}^{2}\right)$ & $117(142)$ & $149(225.5)$ & $117(154)$ & .92 \\
\hline Volume $\left(\mathrm{mm}^{3}\right)$ & $368(538)$ & $423(513)$ & $364(632)$ & .74 \\
\hline Morphologic distortion of adjacent brain parenchyma & & & & $.83^{\mathrm{c}}$ \\
\hline Minimal & $9(14.5)$ & $2(10.0)$ & 7 (16.7) & \\
\hline Mild & $17(27.4)$ & $6(30.0)$ & $11(26.2)$ & \\
\hline Moderate & $24(38.7)$ & $9(45.0)$ & $15(35.7)$ & \\
\hline Severe & $12(19.4)$ & $3(15.0)$ & $9(21.4)$ & \\
\hline $\begin{array}{l}\text { Longest single dimension of distorted parenchyma adjacent to } \\
\text { largest encephalocele (mm) }\end{array}$ & $13(11)$ & $15.5(15)$ & $13(11)$ & .53 \\
\hline Presence of adjacent encephalomalacia & $7(11.3)$ & $0(0.0)$ & $7(16.7)$ & $.09^{\mathrm{c}}$ \\
\hline
\end{tabular}

morphologic distortion within and adjacent to the MCFE was also similar between the seizure and nonseizure groups $(P=.51)$. Among the 13 patients with "severe" brain parenchymal distortion related to the MCFE, 9 were in the nonseizure group and 4 were in the seizure group. The mean longest single dimension of definitively distorted parenchyma within and adjacent to the MCFE was also similar between the seizure $(14 \mathrm{~mm})$ and nonseizure $(13 \mathrm{~mm})$ groups $(P=.54)$. Likewise, the sextant location of the largest MCFE, laterality of the largest MCFE, the total number of MCFEs, the total number of MCF sextants containing encephaloceles, depth and areal extent of the largest MCFE, the presence of CSF-intensity foci within the parenchyma contained in the MCFE, and increased volume of the intracranial subarachnoid space adjacent to the largest MCFE were all similar between the seizure and nonseizure groups.

A separate-though-similar comparison was made between the subset of patients with TLE $(n=20)$ and the nonseizure patient group $(n=42)$. When all the conventional MR imaging characteristics of MCFEs were compared between the patient group with TLE and the patient group without seizures, no significant difference was found (Table 2 and On-line Table 2).

\section{DISCUSSION}

This study found no significant difference between the anatomic MR imaging features of MCFEs found in patients with a history of no seizure, seizure, and TLE. Specifically, we found that MCFE size, location, and number were not predictive of epileptogenicity. Similarly, the presence of encephalomalacia within or adjacent to an MCFE and the subjective degree and measured length of distorted brain parenchyma within and adjacent to an MCFE were not predictive of epileptogenicity. These results suggest that conventional MR imaging features alone may not be useful in distinguishing between MCFEs that are associated with seizure and those that are not associated with seizure.

Recognition of MCFEs has grown during the past decade, raising questions of their clinical significance. While there is some clarity of the role that MCFEs can play in CSF leak, meningitis, and sphenoid sinus encephalocele development, ${ }^{5,16,17}$ the precise etiologic role of MCFEs in epilepsy syndromes has been less clear. For example, Benson et $\mathrm{al}^{3}$ reviewed 203 consecutive internal auditory canal MR imaging examinations and found arachnoid pits in $22 \%$ of scans and MCFEs in 5\% of scans, but none of the patients had a history of seizure. In contrast, Gasparini et $\mathrm{al}^{7}$ looked at the prevalence of MCFE in different groups and found MCFEs in 5/95 (5\%) consecutive patients with TLE of unknown etiology and in $0 \%$ of 150 controls. Additionally, 2 of the patients with TLE in their cohort were seizure-free following resection of their MCFEs. Therefore, while it is probable that MCFEs are the cause of seizure in some patients, it is also likely that MCFEs are more commonly an incidental imaging finding, not associated with seizure. The work presented here attempted to identify conventional MR imaging features of MCFEs that are associated with seizure. We found none.

In some patients with epilepsy, MCFEs can be large or numerous and there may be a great deal of morphologic distortion of the brain parenchyma that extends into the MCFE. In these instances, it may be tempting to assume that the MCFE has some etiologic association with the seizures; however, we 


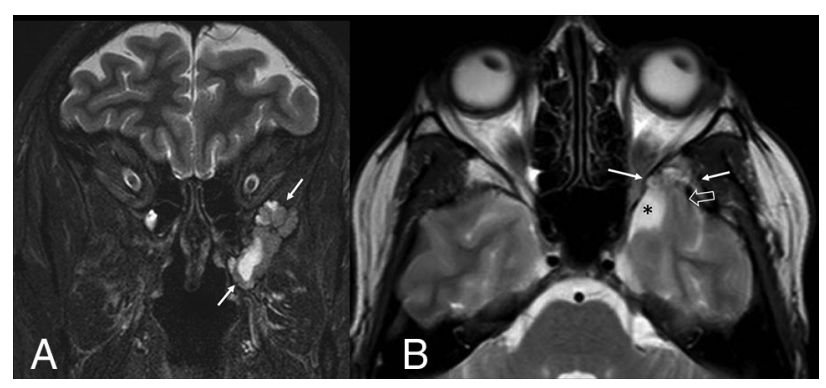

FIG 1. Large left middle cranial fossa encephalocele in a patient with left temporal lobe epilepsy. Coronal STIR image (A, arrows) depicts a focally prominent CSF space and distorted temporal lobe parenchyma surrounded by the scalloped bone margins of the encephalocele. Axial T2 TSE image (B) shows a distorted, stretched appearance of a left temporal lobe gyrus (open arrow) extending into the encephalocele (solid arrows) and asymmetric enlargement of the adjacent intracranial subarachnoid space (asterisk).

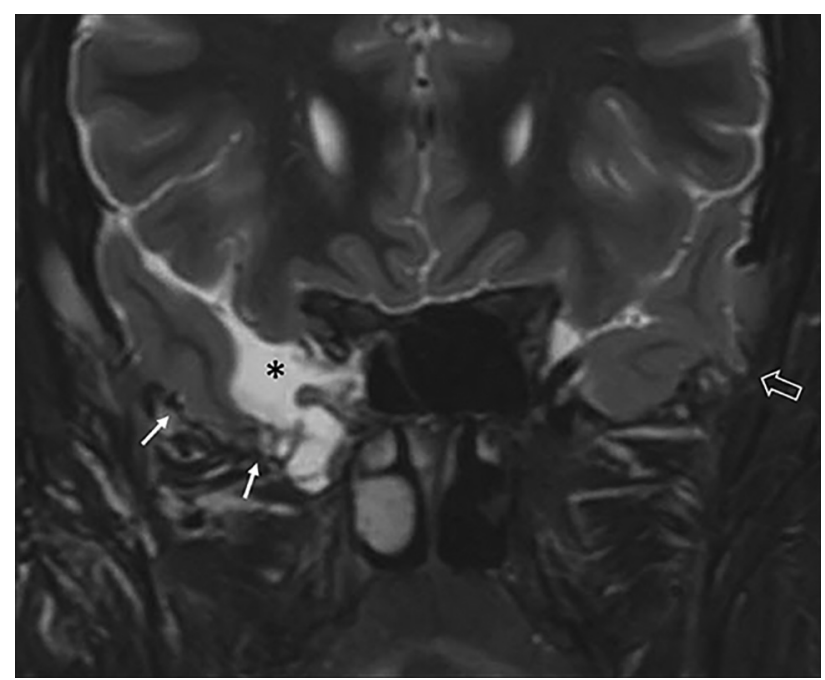

FIG 2. Bilateral middle cranial fossa encephaloceles in a patient with no history of seizure. Coronal STIR image depicts distortion and thinning of temporal lobe parenchyma (solid arrows), which appears adherent to the floor of the encephalocele, and asymmetric enlargement of the adjacent intracranial subarachnoid space (asterisk). A small middle cranial fossa encephalocele is present on the left (open arrow), with associated mild parenchymal distortion.

found no such association. Figures 1 and 2, for example, both depict large MCFEs with associated parenchymal morphologic distortion. Only one of these patients had a history of seizure, and the other did not.

Similarly, it may be tempting to assume that the presence of overt brain parenchymal injury (encephalomalacia) seen in or adjacent to an MCFE would increase the likelihood of epileptogenicity. This is an understandable assumption because neocortical injury due to ischemia is a known risk factor for epilepsy in both pediatric and adult populations. Additionally, the histology of specimens resected from MCFEs in patients who experienced improved seizure frequency following surgery found gliosis to be uniformly present in the MCFE tissue., ${ }^{9,10}$ In our study, the presence of encephalomalacia on MR imaging within or next to MCFEs was not associated with seizure. In fact, encephalomalacia was seen 3 times more frequently in the nonseizure group than in the seizure group, but this difference did not meet statistical significance.

Given the proximity of the temporal lobe to the middle cranial fossa, it is a logical assumption that MCFEs might be more likely to be causative of seizures in patients with TLE than in patients without TLE, and this assumption is supported in the literature. ${ }^{4,8}$ Working from this assumption, we performed an additional analysis comparing the MR imaging features of MCFEs in the nonseizure patient group with those in the TLE group, excluding cases without TLE. In this analysis, we found no significant difference in the conventional MR imaging appearance of MCFEs between the nonseizure and TLE groups.

The findings presented here may be useful during the workup of patients with drug-resistant epilepsy of unclear etiology who are found to have an MCFE and are being considered for intracranial diagnostic or therapeutic procedures. Specifically, assumptions about the likelihood of epileptogenicity of an MCFE should not be based on MCFE size or number, the presence or absence of MCFE-related encephalomalacia, or the degree of brain parenchymal morphologic distortion within and adjacent to the encephalocele. In these cases, other methods for localizing the seizure-onset zone should be used. Toledano et $\mathrm{al}^{4}$ described a typical seizure semiology in patients with left temporal lobe MCFE-related seizures: "significant deterioration of semantic abilities without impairment of the visual perceptual system." For patients with epilepsy presenting with bilateral MCFEs, findings from video-electroencephalography and $\left[{ }^{18} \mathrm{~F}\right]$ FDG-PET have been able to reliably distinguish symptomatic from asymptomatic MCFEs. ${ }^{10}$ Perhaps the most accurate means for distinguishing symptomatic from asymptomatic MCFEs is with intraoperative electrocorticography. Panov et $\mathrm{al}^{9}$ showed that when intraoperative electrocorticography can localize the seizure-onset zone to the region of MCFE, most patients will have a good surgical outcome following resection or detachment of the parenchyma involved with the MCFE. Future MR imaging studies with larger cohorts of patients with TLE may help to elucidate the potential etiologic significance of various anatomic and functional MR imaging characteristics of MCFEs.

For the radiologist who encounters an MCFE during routine clinical practice and may have minimal knowledge of the patient's medical history, the findings of this study may also be useful. Specifically, the conventional MR imaging features of MCFE cannot reliably differentiate symptomatic (seizure-related) from asymptomatic MCFEs. Also, when 1 MCFE is encountered, it may be advisable to search for additional and contralateral encephaloceles. In our study, $66 \%$ of patients with MCFEs had $\geq 2$ MCFEs, and MCFEs were seen bilaterally in $51 \%$ of cases. Corroborating the recent work of Benson et $\mathrm{al}^{3}{ }^{3}$ our study shows that MCFEs can be incidentally discovered findings on $\mathrm{MR}$ imaging performed in patients with no history of seizure.

Although not the subject of the study presented here, MCFEs and MCF arachnoid pits have known associations with disease processes other than epilepsy. When an MCFE is encountered in clinical practice, radiologists may be able to add diagnostic value by looking for clues to these other associated diseases. For example, CSF leak from MCF defects that involve either the mastoid 
portion of the temporal bone or the lateral recess of the sphenoid sinus can result in fluid opacification of those structures, diffuse dural thickening/contrast enhancement from low intracranial pressure, and imaging findings of meningitis. Idiopathic intracranial hypertension also has an association with MCFEs and arachnoid pits and has associated imaging findings including an enlarged and empty sella and stenosis of the lateral portions of the transverse sinuses and protruding optic discs. In our study, there was a slight female preponderance (64\%) of patients with MCFE, which is of uncertain clinical significance but may support an association between MCFE and intracranial hypertension.

Much is still unknown about the pathophysiology of encephaloceles. Some clearly have a congenital origin related to genetic disease (eg, occipital encephalocele in Meckel-Gruber syndrome) ${ }^{18}$ or problems with closure of the anterior neural pore (eg, frontonasal encephalocele). ${ }^{19,20}$ Other encephaloceles are acquired, arising at the site of skull base fractures or a skull base operation. ${ }^{2}$ However, in the authors' experience, most MCFEs encountered in neuroradiology practice are unrelated to congenital, traumatic, or surgical etiologies and may, instead, fall into the "spontaneous" category. Spontaneous MCFEs appear to be associated with chronically elevated intracranial pressure in a proportion of cases. ${ }^{2,21}$ The MR imaging features described here may provide some clues to their pathophysiology. Some degree of gross parenchymal morphologic distortion was present in all cases, and a subjective asymmetric increase of the intracranial subarachnoid space adjacent to the largest MCFE was present in 38\% of patients. Larger MCFEs allowed better depiction of the parenchymal distortion, and a common finding in those cases was parenchymal thinning as it extended into the MCF. The distorted parenchyma was closely approximated with the margins of the MCF defect (Figs 1 and 2).

One possible mechanism to account for these findings is that the brain is somehow drawn into an aberrant arachnoid granulation in the MCF, the pia becomes adherent to the margins of the MCFE, and the displacement of brain parenchyma into the MCFE gives it a stretched appearance and results in ex vacuo enlargement of the adjacent intracranial subarachnoid space. Age-related global cerebral atrophy would accentuate some of these findings in which the cerebrum retracts away from the inner table of the calvaria as it shrinks, leading to further thinning of the pedicle of cerebral tissue extending into the MCFE. Similar imaging findings are seen in association with aberrant arachnoid granulations in the posterior fossa bone and transverse sinuses that can entrain and stretch the cerebellar parenchyma. ${ }^{22}$ However, there are a few reports of cortical microdysgenesis and laminar disorganization of specimens resected from MCFEs, ${ }^{10,13,23,24}$ which confuse the hypothesis that MCFEs are an acquired phenomenon.

Encephalomalacia of the brain within or immediately adjacent to the MCFE was seen in $12 \%$ of patients in this study. A few mechanisms might explain this finding, including local mechanical trauma related to the apparently tethered brain within the MCFE, sequela of prior blunt trauma with head injury, or local gliosis from chronic seizures. Stippled or punctate foci of CSF-intensity T2 signal within the parenchyma located in the MCFE were observed in $53 \%$ of our patients and have been described by others. ${ }^{23}$ These had an appearance suggestive of clustered dilated perivascular spaces or other CSF-filled parenchymal clefts, but their etiology was unclear. Notably, this finding was distinct from the confluent regions of T2-hyperintense signal seen with cases of encephalomalacia.

The prospective accrual of patients with MCFEs during routine clinical practice of neuroradiology was a strength of this study in that it formed a patient cohort somewhat representative of cases that might be encountered by practicing neuroradiologists. Many cases of MCFE were undoubtedly missed during the accrual period, however, because clinical practice can be busy, leading to oversight of these often small, inconspicuous lesions. Additionally, there was great variation in the MR imaging protocols included in the study. Another shortcoming of this study was the variability of patient medical histories available for review, leading to possible erroneous assignment of patients with an actual history of seizure to the nonseizure group. It is likely that the MCFEs present in many of the patients in the seizure group were not at all related to their seizures and were instead an unrelated coincidental finding. Indeed, 76\% (26/35) of patients in the seizure group were found to have risk factors for seizure other than MCFEs. A larger, case-control study including only patients with epilepsy with definitively epileptogenic MCFEs, confirmed by intracranial monitoring or postsurgical seizure freedom, may have provided a better chance for uncovering etiologically significant MR imaging features of MCFEs. Such a study would be challenging to build due to the paucity of these cases.

\section{CONCLUSIONS}

Anatomic MR imaging features alone may not be useful in distinguishing MCFEs associated with seizure from MCFEs not associated with seizure. MCFEs that are large or numerous, MCFEs associated with encephalomalacia, and MCFEs associated with severe morphologic distortion of adjacent brain parenchyma can be seen with similar frequency between patients with and without a history of seizures.

\section{ACKNOWLEDGMENTS}

The authors would like to acknowledge Priya Srikanth, MPH, for her thoughtful contributions to the statistical analysis.

Disclosures: Brian D. Clark-UNRELATED: Employment: TRG Medical Imaging, Comments: employed as a radiologist at TRG private practice since August 2019. David C. Spencer-UNRELATED: Board Membership: American Epilepsy Society, Comments: unpaid; Employment: Oregon Health \& Science University, VA Portland Health Care System, Comments: salary for my work as a neurologist; Expert Testimony: various, Comments: rare review of legal cases, 1 deposition in last 3 years; Grants/Grants Pending: Eisai Co, Engage Therapeutics, Comments: clinical studies*; Royalties: Oxford University Press, Comments: small amount of royalties for authoring a book several years ago; Payment for Development of Educational Presentations: Neurology Residents Scholar Program, Comments: co-chair of this educational program for residents. *Money paid to the institution.

\section{REFERENCES}

1. Byrne RW, Smith AP, Roh D, et al. Occult middle fossa encephaloceles in patients with temporal lobe epilepsy. World Neurosurg 2010;73:541-46 CrossRef Medline

2. Carlson ML, Copeland WR, 3rd, Driscoll CL, et al. Temporal bone encephalocele and cerebrospinal fluid fistula repair utilizing the middle cranial fossa or combined mastoid-middle cranial fossa approach. J Neurosurg 2013;119:1314-22 CrossRef Medline 
3. Benson JC, Lane J, Geske JR, et al. Prevalence of asymptomatic middle cranial fossa floor pits and encephaloceles on MR imaging. AJNR Am J Neuroradiol 2019;40:2090-93 CrossRef Medline

4. Toledano R, Jimenez-Huete A, Campo P, et al. Small temporal pole encephalocele: a hidden cause of "normal" MRI temporal lobe epilepsy. Epilepsia 2016;57:841-51 CrossRef Medline

5. Schuknecht B, Simmen D, Briner HR, et al. Nontraumatic skull base defects with spontaneous CSF rhinorrhea and arachnoid herniation: imaging findings and correlation with endoscopic sinus surgery in 27 patients. AJNR Am J Neuroradiol 2008;29:54249 CrossRef

6. Hammer A, Baer I, Geletneky K, et al. Cerebrospinal fluid rhinorrhea and seizure caused by temporo-sphenoidal encephalocele. $J$ Korean Neurosurg Soc 2015;57:298-302 CrossRef Medline

7. Gasparini S, Ferlazzo E, Pustorino G, et al. Epileptogenic role of occult temporal encephalomeningocele: case-control study. Neurology 2018;90:e1200-03 CrossRef Medline

8. Campbell ZM, Hyer JM, Lauzon S, et al. Detection and characteristics of temporal encephaloceles in patients with refractory epilepsy. AJNR Am J Neuroradiol 2018;39:1468-72 CrossRef Medline

9. Panov F, Li Y, Chang EF, et al. Epilepsy with temporal encephalocele: characteristics of electrocorticography and surgical outcome. Epilepsia 2016;57:e33-38 CrossRef Medline

10. Saavalainen T, Jutila L, Mervaala E, et al. Temporal anteroinferior encephalocele: an underrecognized etiology of temporal lobe epilepsy? Neurology 2015;85:1467-74 CrossRef Medline

11. Shimada S, Kunii N, Kawai K, et al. Spontaneous temporal pole encephalocele presenting with epilepsy: report of two cases. World Neurosurg 2015;84:e861.e1-6 CrossRef Medline

12. Abou-Hamden A, Lau M, Fabinyi G, et al. Small temporal pole encephaloceles: a treatable cause of "lesion negative" temporal lobe epilepsy. Epilepsia 2010;51:2199-02 CrossRef Medline

13. Leblanc R, Tampieri D, Robitaille $Y$, et al. Developmental anterobasal temporal encephalocele and temporal lobe epilepsy. I Neurosurg 1991;74:933-39 CrossRef Medline
14. Morone PJ, Sweeney AD, Carlson ML, et al. Temporal lobe encephaloceles: a potentially curable cause of seizures. Otol Neurotol 2015;36:1439-42 CrossRef Medline

15. Fisher RS, Cross JH, French JA, et al. Operational classification of seizure types by the International League Against Epilepsy: Position Paper of the ILAE Commission for Classification and Terminology. Epilepsia 2017;58:522-30 CrossRef Medline

16. Settecase F, Harnsberger HR, Michel MA, et al. Spontaneous lateral sphenoid cephaloceles: anatomic factors contributing to pathogenesis and proposed classification. AJNR Am J Neuroradiol 2014;35:78489 CrossRef Medline

17. Shetty PG, Shroff MM, Fatterpekar GM, et al. A retrospective analysis of spontaneous sphenoid sinus fistula: MR and CT findings. AJNR Am J Neuroradiol 2000;21:337-42 Medline

18. Parelkar SV, Kapadnis SP, Sanghvi BV, et al. Meckel-Gruber syndrome: A rare and lethal anomaly with review of literature. J Pediatr Neurosci 2013;8:154-57 CrossRef Medline

19. Barkovich AJ, Vandermarck P, Edwards MS, et al. Congenital nasal masses: CT and MR imaging features in 16 cases. AJNR Am J Neuroradiol 1991;12:105-16 Medline

20. Rojas L, Melvin JJ, Faerber EN, et al. Anterior encephalocele associated with subependymal nodular heterotopia, cortical dysplasia and epilepsy: case report and review of the literature. Eur J Paediatr Neurol 2006;10:227-29 CrossRef Medline

21. Watane GV, Patel B, Brown D, et al. The significance of arachnoid granulation in patients with idiopathic intracranial hypertension. $J$ Comput Assist Tomogr 2018;42:282-85 CrossRef Medline

22. Battal B, Castillo M. Brain herniations into the dural venous sinuses or calvarium: MRI of a recently recognized entity. Neuroradiol J 2014;27:55-62 CrossRef Medline

23. Gasparini S, Ferlazzo E, Villani F, et al. Refractory epilepsy and encephalocele: lesionectomy or tailored surgery? Seizure 2014;23:58384 CrossRef Medline

24. Giulioni M, Licchetta L, Bisulli F, et al. Tailored surgery for drug-resistant epilepsy due to temporal pole encephalocele and microdysgenesis. Seizure 2014;23:164-66 CrossRef Medline 